\title{
Symptoms of Depression in Patients with Chemosensory Disorders
}

\author{
Ben Chen $^{a, b}$ Cara Benzien $^{a} \quad V^{2}$ anda Faria ${ }^{a, c, d}$ Yuping Ning ${ }^{b}$ Mandy Cuevas $^{a}$ \\ Jana Linke ${ }^{a}$ llona Croye Antje Haehner ${ }^{\mathrm{a}}$ Thomas Hummel $^{\mathrm{a}}$ \\ aDepartment of Otorhinolaryngology, Smell \& Taste Clinic, TU Dresden, Dresden, Germany; ${ }^{\text {bDepartment of }}$ \\ Psychiatry, The Affiliated Brain Hospital of Guangzhou Medical University (Guangzhou Huiai Hospital), \\ Guangzhou, China; 'Department of Psychology, Uppsala University, Uppsala, Sweden; dDepartment of Anesthesiology, \\ Center for Pain and the Brain, Perioperative and Pain Medicine, Boston Children's Hospital, Harvard Medical School, \\ Boston, MA, USA; 'Department of Psychosomatic Medicine, TU Dresden, Dresden, Germany
}

\section{Keywords \\ Nose $\cdot$ Olfaction $\cdot$ Anosmia $\cdot$ Smell $\cdot$ Depression $\cdot$ Emotion}

\begin{abstract}
Introduction: Patients with chemosensory dysfunction frequently report symptoms of depression. The current study aims to clarify whether the type (smell dysfunction, taste dysfunction, and mixed smell and taste dysfunction), severity, duration, or cause of dysfunction have differential impacts on the symptoms of depression. Methods: 899 patients with chemosensory disorders and 62 controls were included. Following a structured interview and an otorhinolaryngological examination, subjects underwent olfactory tests (Sniffin' Sticks), gustatory tests (taste sprays) and an assessment of depressive symptoms (Beck Depression Inventory). Information on the cause and duration of disorders was also collected. Results: Patients with combined olfactory/gustatory dysfunction had higher depression scores than patients with smell dysfunction only and controls, and no significant difference was found between the smell dysfunction and controls. Anosmia patients, but not hyposmia patients, exhibited higher depression scores than controls. Among various causes of chemosensory disorders, patients
\end{abstract}

from the posttraumatic group had higher depression scores than patients with other causes of chemosensory dysfunction (sinonasal, idiopathic, or postinfectious). Multiple linear regression analyses suggested that reduced olfactory function was associated with enhanced depression scores in the olfactory disorders group $(B=-0.326, t=-2.294$, and $p=$ $0.02)$ and in all patients with chemosensory disorders $(B=$ $-0.374, t=-2.550, p=0.017)$. Discussion/Conclusion: Simultaneously decreased input of olfaction and gustation seems to have an additive effect on the exacerbation of emotional dysfunction. Early intervention should be considered for depression symptoms in patients with mixed olfactory/gustatory dysfunction in clinical practice. @ 2021 S. Karger AG, Basel

\section{Introduction}

Chemosensory disorders include olfactory disorders and taste dysfunctions, and such disorders can not only impair quality of life (QoL) but also lead to emotional dysfunction $[1,2]$. Depressive symptoms are not uncommon in patients with chemosensory disorders [3-5]. Croy et al. [1] reported that approximately $1 / 4-1 / 3$ of patients

\footnotetext{
Ben Chen

Department of Otorhinolaryngology, Smell \& Taste Clinic, TU Dresden Fetscherstraße 74

DE-01307 Dresden (Germany)

chenbenpielo@sina.com
} 
with olfactory disorders exhibit depressive symptoms. Several studies indicate positive correlations between olfactory deficits and depressive symptoms [6-9], but these correlation coefficients tend to be weak. Compared with olfactory disorders, few studies have focused on depressive symptoms in patients with gustatory disorder. Limited evidence suggests that patients with gustatory disorder have higher depression scores, and the proportion of reporting depressive symptoms ranges from 25 to $36 \%$ [10-12]. Hence, depression is an important symptom of chemosensory disorders, and a lack of input of smell and taste may result in emotional dysfunction.

A growing body of research supports the existence of a close relationship between olfaction and depression [13-16], and many brain areas associated with the processing of emotions overlap with the olfactory pathway, such as the amygdala, hippocampus, insular and orbitofrontal cortex [17]. Previous studies have suggested that patients with depression exhibit olfactory deficits [17-20] and many structural and functional abnormalities in the olfactory pathway $[21,22]$. Additionally, depressive symptoms are associated with reduced odor identification $[22,23]$. Systematic exposure to odors, so-called olfactory training, may improve depressive symptoms [23, 24 ], suggesting that enhancement of olfactory input may be helpful in emotional regulation. Compared with olfactory disorders, fewer studies have focused on gustatory function in patients with depression. Limited evidence points toward a relation between taste deficits and depression, but their results are somewhat contradictory and inconclusive. One study suggested that sweet taste sensitivity was influenced by 5-HTTLPR genotype and affected by seasonal affective disorder [25]. Another study failed to prove the association between winter depression and alterations in gustatory function [26].

Interestingly, the reciprocal interaction between chemosensory change and emotion has been repeatedly reported $[27,28]$. (1) Chemosensory disorders can lead to depression. First, olfactory impairment may lead to restrictions in olfactory-related areas, which can affect QoL and in turn may enhance the likelihood of depression. Second, olfactory loss may reduce input from the olfactory bulb via the amygdala into the limbic system and insula and then cause dysfunction of convergence and salience processes, resulting in functional abnormalities of the brain. (2) Depression may also affect chemosensory function. On the one hand, reduced attention and reduced turnover rate of olfactory receptor neurons in the olfactory epithelium may cause reduced olfactory function in depression. These effects are reduced after remis- sion of depression. On the other hand, decreased volume of olfactory bulb in depression patients may lead to reduced signaling from OB to central olfactory areas and exacerbate depressive symptoms.

Taken together, olfactory and gustatory input plays an important role in the activity of limbic circuits, and the lack of such input in the case of chemosensory disorders may cause depression. Although early studies commonly included patients with smell dysfunction and patients with taste dysfunctions, few studies have included patients with mixed olfactory/gustatory dysfunction, which is a common patient population in clinical practice. The depression status in patients with mixed olfactory/gustatory dysfunction remains unclear. Recent work suggests that combined sensory impairments may result in reduced QoL, suggesting that the reduced input from various sensory channels may have an additive effect [29]. However, there was no assessment of depression in this study. Therefore, we hypothesized that simultaneous decreases in the olfactory and gustatory senses may exacerbate emotional dysfunction, and patients with mixed olfactory/gustatory dysfunction may have more severe depressive symptoms than patients with only one of these disorders. Additionally, because of the decreased olfactory input, patients with anosmia may have more obvious depressive symptoms than patients with hyposmia and controls. Moreover, since different causes of chemosensory disorders may have various effects on QoL [1], we assumed that they may also exert different influences on depression. The present study aimed to compare the depressive symptoms in patients with mixed olfactory/gustatory dysfunction and purely olfactory disorder and gustatory disorder and to explore the associations between chemosensory function and depressive symptoms in different groups.

\section{Method}

\section{Subjects}

The current study retrospectively included 961 subjects (588 males and 373 females) at the Smell and Taste Clinic at the Department of Otorhinolaryngology of the TU Dresden, Germany. They consisted of 2 groups: patients with chemosensory disorder $(n=$ 899; 547 male, 352 female; age $55.4 \pm 13.3$ years) and normosmic/ normogeusic controls ( $n=62 ; 41$ male, 21 female; age $46.8 \pm 13.6$ years). All subjects received a detailed otorhinolaryngological examination including nasal endoscopy, a structured history, and a standardized test for smell and taste function, and the diagnoses of chemosensory disorders were confirmed by an ENT specialist. We included subjects presenting themselves to the Smell and Taste Clinic between February 1998 and August 2017 who had filled in the Beck Depression Inventory (BDI). The exclusion criteria in- 
volved major diseases (e.g., complicated diabetes, kidney disease, severe neurological and psychiatric diseases), pregnancy, or lactation. In essence, we included subjects who were not seriously ill, apart from the chemosensory disorder. In all included subjects, information was obtained on the cause and duration of the disorder [30], and symptoms of depression were assessed using the German version of the BDI. The standard cutoff scores for the BDI were as follows: 0-9: minimal depression; 10-18: mild depression; 19-29: moderate depression; 30-63: severe depression [31].

All subjects received a detailed otorhinolaryngological examination including nasal endoscopy; a structured history was taken; and all subjects underwent standardized tests for smell and taste function [32]. The cause of chemosensory disorder was confirmed by an otolaryngologist after overall consideration of the patients' disease history (which is especially important in dysgeusia patients), otorhinolaryngological examination, and neuroimaging test. Controls did not exhibit major otorhinolaryngology disorders; they did not mention any disturbances of smell or taste. Parosmia was defined as the perception of distorted odors in the presence of an odor, that is, the parosmic impression was not present/ largely reduced when clamping the nose. Phantosmia was defined as the perception of an odor in the absence of an odor source [30].

This retrospective study was conducted in accordance with the guidelines of the Declaration of Helsinki of 1975 (revised in 1983), and all procedures were approved by the Ethics Committee at the University Hospital of the TU Dresden (protocol number EK251112006). As this is a retrospective study and patient data were completely anonymized/de-identified, informed consent was not obtained.

\section{Assessment of Taste Function - "Taste Sprays"}

Gustatory function was screened with suprathreshold tastants sprayed onto the tongue. So-called "taste sprays" had to be identified as sweet, sour, salty, or bitter [32]. Taste spray was assessed by the 4 basic tastes at suprathreshold concentrations (sweet: sucrose, 1.5 g; sour: citric acid, 0.75 g; salty: sodium chloride, $1.125 \mathrm{~g}$; and bitter: quinine hydrochloride, $7.5 \mu \mathrm{g}$; all tastants were dissolved in $15 \mathrm{~g}$ of water). The tastants were sprayed on the middle of the tongue of the participants (approximately $0.1 \mathrm{~mL}$ per spray), and the participants were then asked to identify the taste according to a list with the 4 taste descriptors. After each sample, subjects rinsed with water. The number of correctly identified tastes was summed up to a taste score. Scores range from 0 to 4.

\section{Assessment of Olfactory Function - "Sniffin Sticks"}

Orthonasal olfactory function was measured by means of the "Sniffin Sticks" test, which is based on odor-containing felt-tip pens [33]. This test consists of 3 subtests: threshold, discrimination, and identification test. For each subtest, the pen's cap was removed, and its felt tip was presented approximately $2 \mathrm{~cm}$ in front of both nostrils of the subject for approximately $3 \mathrm{~s}$. The testing procedure began with the threshold part in a triple-forced-choice paradigm where subjects had to discriminate the odor (phenyl ethyl alcohol) from 2 blanks (filled with solvent propylene glycol). Starting with the lowest phenyl ethyl alcohol concentration, a staircase paradigm was used where 2 correct or 1 incorrect answers led to a decrease or increase in concentration, the so-called turning point. The resulting threshold score was the mean of the last 4 turning points in the staircase (range from 1 to 16). The next subtest performed was the discrimination test, where 2 pens had the same odor while the other 1 had a different scent, which had to be identified (range from 0 to 16). The last task was the identification test, where the subject was asked to choose the object that describes the odor the best using a 4-alternative forced choice from flash cards that had both the picture and name of the object (range from 0 to 16). The scores of the olfactory subtests were then summed to build the overall TDI (threshold, discrimination, and identification) score (range from 1 to 48). The cutoff score for the TDI is 30.75 between normal olfaction and hyposmia and 16 between hyposmia and anosmia [34, 35].

\section{Statistics}

Statistical Package for Social Sciences version 22.0 (IBM SPSS 22.0, Chicago, IL, USA) was used for the statistical analyses. Differences in relation to age and duration of disorder were evaluated with one-way ANOVAs. Differences in olfactory scores, depression scores, and taste scores were evaluated with ANCOVA, and control variables included age and sex. Post hoc least significant difference tests were used for multiple comparisons. Spearman's correlations were calculated to assess associations between depression and age or duration of disorder, and partial correlation analyses were used to analyze the associations between depression and other indexes. Control variables included age and sex. Stepwise multiple linear regression analysis was used to analyze the effect of different variables on depressive scores, including age, sex, TDI scores, taste scores, duration of disorders, BMI, and disease causes. The significance level for inclusion was set at $p<0.05$, whereas the significance level for exclusion was set at $p>0.10$.

\section{Results}

\section{Demographics}

From a sample consisting of 899 patients with chemosensory disorders, 804 were diagnosed with olfactory disorders only, 47 were diagnosed with gustatory disorders only, and 48 patients were diagnosed with mixed olfactory/gustatory dysfunction. Among patients with olfactory disorders, 473 patients were diagnosed as hyposmic, 379 patients as functionally anosmic (further termed anosmic), and 245 patients had accompanying parosmia or phantosmia (177 hyposmia and 68 anosmia). Among patients with gustatory disorders, 93 patients were diagnosed as hypogeusic, 1 patient as ageusic, and 1 patient was diagnosed as purely parageusic, and no patients with hypogeusia and ageusia were accompanied by parageusia (A partial loss of taste function is defined as hypogeusia and a complete loss is ageusia. Parageusia is a distorted perception of particular tastes).

\section{Comparison of Different Kinds of Chemosensory \\ Disorders and Controls}

Demographic data, olfactory scores, and taste scores are listed for all the groups in Table 1. Among different 


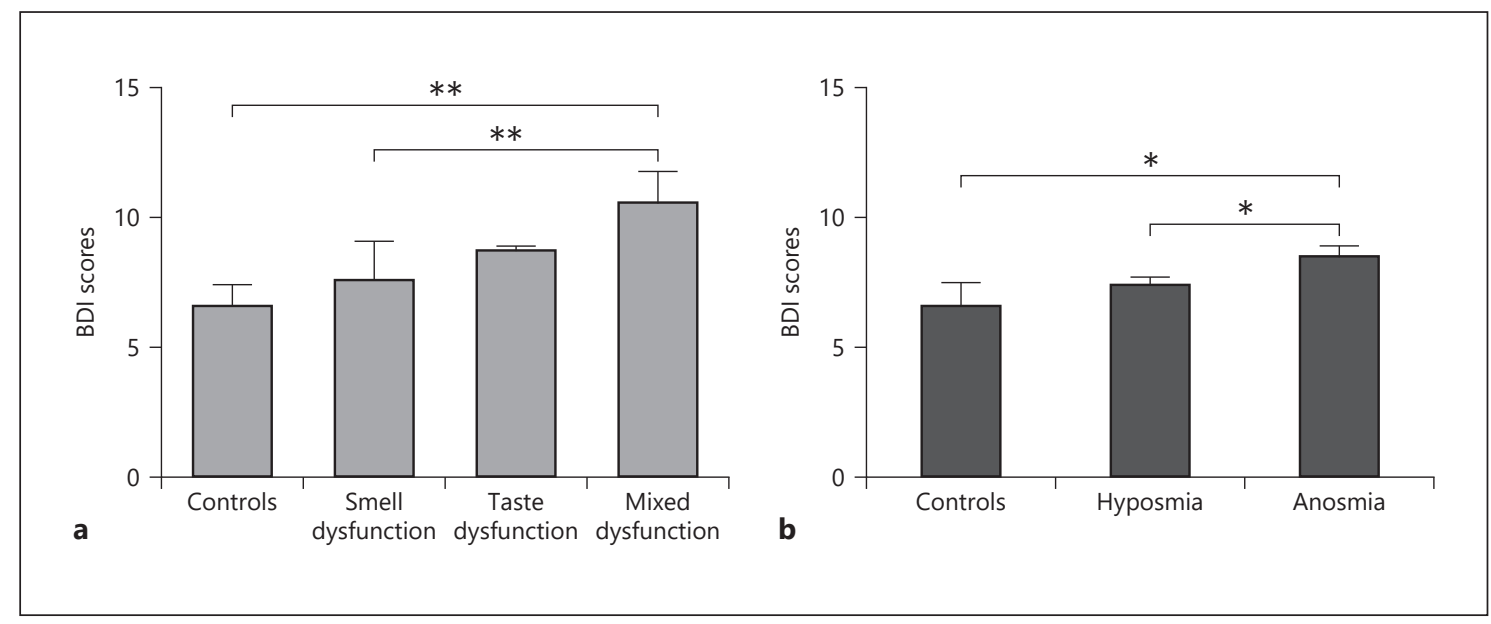

Fig. 1. a BDI scores in patients with chemosensory disorders and controls. b BDI scores in healthy controls, hyposmia, and anosmia. Error bars represent means $\pm \mathrm{SD}^{*} p<0.05,{ }^{* *} p<0.01,{ }^{* * *} p<0.001$. BDI, Beck Depression Inventory.

Table 1. Demographics, clinical characteristics of the participants, and comparison among chemosensory disorders

\begin{tabular}{|c|c|c|c|c|c|c|c|c|}
\hline & $\begin{array}{l}\text { Healthy } \\
\text { control } \\
(n=62)\end{array}$ & $\begin{array}{l}\text { Smell } \\
\text { dysfunction } \\
(n=804)\end{array}$ & $\begin{array}{l}\text { Taste } \\
\text { dysfunction } \\
(n=47)\end{array}$ & $\begin{array}{l}\text { Mixed taste and } \\
\text { smell dysfunction } \\
(n=48)\end{array}$ & $F / \chi^{2}$ & $p$ value & $\eta^{2}$ & Post hoc \\
\hline Sex (male/memale) & $41 / 21$ & $491 / 313$ & $29 / 18$ & $27 / 21$ & 0.990 & 0.80 & - & - \\
\hline Duration (months) & - & $160.1 \pm 90.7$ & $121.7 \pm 67.0$ & $130.1 \pm 84.8$ & 6.320 & 0.002 & 0.017 & $\mathrm{~B}>\mathrm{C}, \mathrm{D}$ \\
\hline BDI* & $6.6 \pm 6.0$ & $7.6 \pm 7.0$ & $8.7 \pm 10.0$ & $10.6 \pm 8.5$ & 4.756 & 0.024 & 0.024 & $\mathrm{~A}, \mathrm{C}<\mathrm{D}$ \\
\hline Moderate depression (BDI 19-29) & $1(1.6 \%)$ & $46(5.7 \%)$ & $2(4.3 \%)$ & $5(10.4 \%)$ & - & - & & - \\
\hline Severe depression $(\mathrm{BDI}>30)$ & $1(1.6 \%)$ & $14(1.7 \%)$ & $3(6.4 \%)$ & $2(4.2 \%)$ & - & - & & - \\
\hline Odor identification* & $12.8 \pm 2.2$ & $7.0 \pm 3.5$ & $12.6 \pm 2.4$ & $7.4 \pm 3.6$ & 43.438 & $<0.001$ & 0.192 & $\mathrm{~A}, \mathrm{C}>\mathrm{B}, \mathrm{D}$ \\
\hline Odor discrimination* & $12.7 \pm 2.0$ & $8.1 \pm 3.1$ & $12.3 \pm 2.3$ & $7.4 \pm 3.1$ & 32.025 & $<0.001$ & 0.153 & $\mathrm{~A}, \mathrm{C}>\mathrm{B}, \mathrm{D}$ \\
\hline Odor threshold* & $8.3 \pm 2.8$ & $3.1 \pm 2.8$ & $7.5 \pm 2.8$ & $2.9 \pm 3.0$ & 43.493 & $<0.001$ & 0.195 & $\mathrm{~A}, \mathrm{C}>\mathrm{B}, \mathrm{D}$ \\
\hline TDI score* & $33.9 \pm 5.0$ & $18.2+7.8$ & $31.8 \pm 4.8$ & $17.5 \pm 7.9$ & 54.602 & $<0.001$ & 0.233 & $\mathrm{~A}, \mathrm{C}>\mathrm{B}, \mathrm{D}$ \\
\hline
\end{tabular}

BDI, Beck Depression Inventory. * ANCOVA were used; covariates were age and sex. For the post hoc tests, A stands for healthy control, B for smell dysfunction, $\mathrm{C}$ for taste dysfunction, and $\mathrm{D}$ for mixed disorder.

types of chemosensory disorders, patients with mixed dysfunction had higher depressive scores than controls $(p=0.032)$ and patients with smell dysfunction $(p=$ $0.033)$ but not patients with taste dysfunctions $(p=0.567)$ $(F=4.76, p<0.001$, and df $=5)($ Fig. 1a; Table 1). Women had significantly higher depression scores than men $(t=3.56, p<0.001)$. Divided by severity of depressive symptoms, the distribution of depressive level in various groups was significantly different $\left(\chi^{2}=17.8, p=0.037\right)$, and patients with mixed dysfunction exhibited higher proportions of mild and moderate depression than the other groups (Table 1).

\section{Comparison of Controls, Hyposmia, and Anosmia}

Patients with anosmia had higher BDI scores than controls $(p=0.023)$ and patients with hyposmia ( $p=$ 0.039 ) (Fig. 1b). No significant difference was found between patients with hyposmia and controls $(p=0.684)$. 
Fig. 2. BDI scores in different causes of olfactory dysfunction and controls. Error bars represent means \pm SD ${ }^{*} p<0.05,{ }^{* *} p<$ $0.01,{ }^{* * *} p<0.001$. BDI, Beck Depression Inventory.

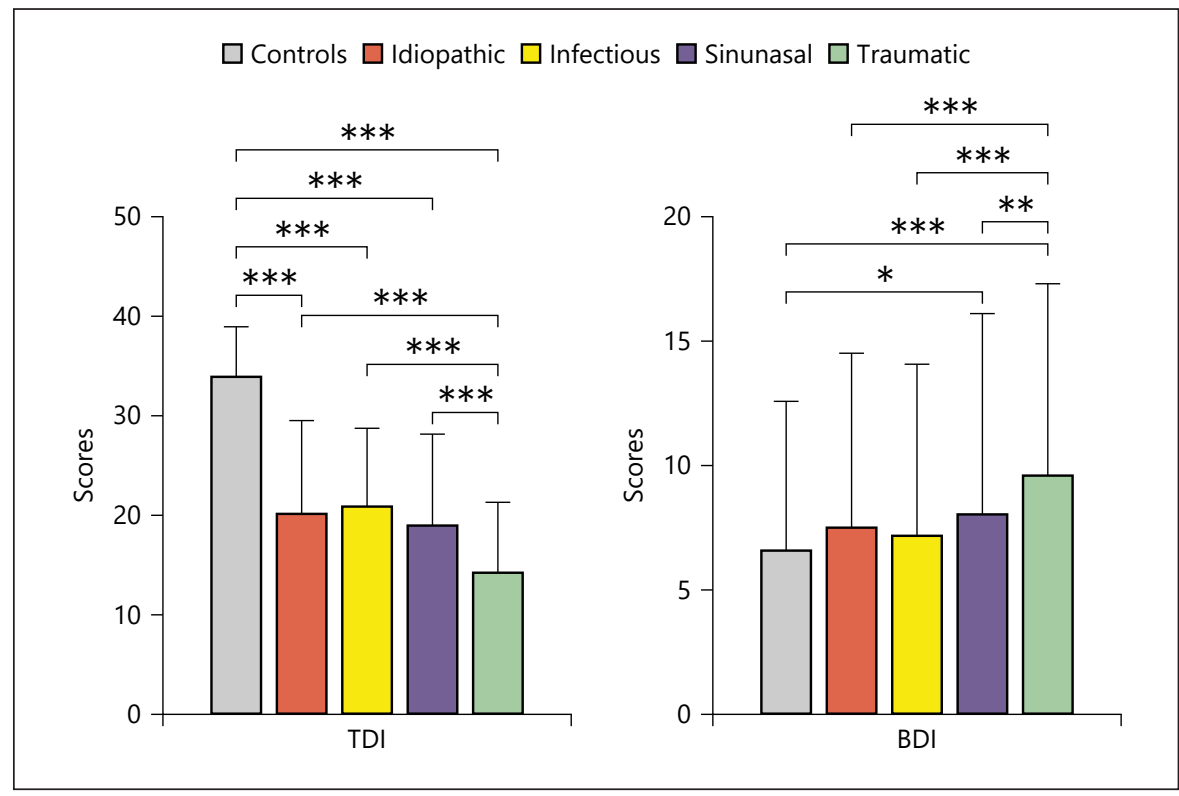

\section{Comparison of Different Causes of Chemosensory} Disorders and Controls

With regard to causes of olfactory dysfunction, there were 142 patients caused by sinonasal diseases, 184 by idiopathic reasons, 336 by infection, 134 by trauma, and 40 by other reasons (including congenital, neurodegeneration, and dental treatment). With regard to causes of gustatory dysfunction, there were 6 patients caused by trauma, 5 by toxic, 10 by tonsillectomy, 6 by sinonasal diseases, 20 by infection, 41 by idiopathic, and 7 by burning mouth syndrome.

Across different disease causes of olfactory dysfunction, the sinonasal group and posttraumatic group exhibited higher BDI scores than the controls. Moreover, the posttraumatic group had significantly higher BDI scores and lower TDI scores than the other groups (Fig. 2).

\section{Association between Olfactory/Gustatory Function and Factors}

In correlation analyses across all subjects and patients with chemosensory disorders, the depression score was negatively correlated with odor scores (association between BDI and TDI scores: $r=-0.11, p=0.001 ; r=-0.14$, $p=0.001)$. In patients with smell dysfunction, depression scores were negatively correlated with odor threshold $(r=-0.10, p=0.010)$, odor discrimination $(r=-0.10, p=$ $0.007)$, and TDI scores $(r=-0.10, p=0.008)$; no such correlation was found in patients with taste dysfunction and mixed dysfunctions (see online suppl. Table 1; see www.

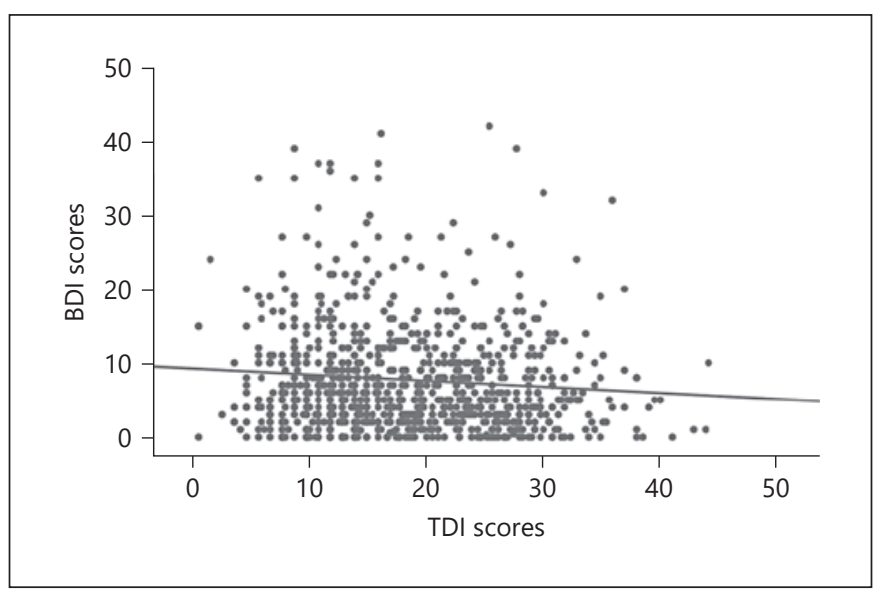

Fig. 3. Correlation between BDI scores and TDI scores in chemosensory disorders. BDI, Beck Depression Inventory.

karger.com/doi/10.1159/000513751for all online suppl. material).

Regression analyses suggested that TDI scores were the only variable associated with BDI scores in the smell dysfunction group $(B=-0.326, \beta=-0.398, t=-2.294$, $p=0.029,95 \%$ CI $[-0.618,-0.035]$, and $\left.R^{2}=0.194\right)$ and all patients with chemosensory disorders $(B=-0.374, \beta=$ $-0.441, t=-2.550, p=0.017,95 \%$ CI $[-0.675,-0.073]$, and $R^{2}=0.194$ ) (Fig. 3), and no significant variable entered the model in the taste dysfunctions group and mixed dysfunctions group. 


\section{Discussion}

This is the first study to analyze symptoms of depression in patients with mixed chemosensory disorders. The data revealed 4 major results. (1) Patients with mixed taste and smell dysfunction have higher depression scores than patients with smell dysfunction and controls. (2) Anosmia patients, but not hyposmia patients, had higher depression scores than controls. (3) Among the different causes of chemosensory disorders, the posttraumatic group had higher depression scores than the other groups. (4) TDI scores were significantly associated with depression scores in patients with smell dysfunction and combined chemosensory disorders.

Current results demonstrated that patients with mixed dysfunctions tended to exhibit more severe symptoms of depression compared with controls and patients with smell dysfunction. On average, patients with mixed dysfunction also had higher BDI scores than patients with taste dysfunctions, but this difference did not reach statistical significance. This may partly be due to the smaller sample size of patients with taste dysfunction compared with smell dysfunction patients. However, our sample size comprised a minimum of approximately 50 individuals in each group, which constitutes a relatively large clinical sample, especially with regard to patients with taste dysfunction. On the one hand, as mentioned previously, chemosensory input can influence emotional processing. Patients with chemosensory disorders seem to be vulnerable to depression [36], and systematic, regular exposure to odors ("olfactory training") may improve depressive symptoms [23]. On the other hand, emotional dysfunction can lead to chemosensory disorders. Patients with depression often exhibit chemosensory dysfunction and relevant brain abnormalities, and the remission of depression results in the recovery of chemosensory dysfunction $[22,25]$. Therefore, we assume that simultaneously decreased input of olfaction and gustation has additive effects on the exacerbation of emotional dysfunction, resulting in a higher proportion of mild and moderate depression. It can also be assumed that there may be more functional abnormalities in the common brain areas of emotional processing and chemosensory pathways in patients with mixed dysfunctions (such as the amygdala, hippocampus, insular and orbitofrontal cortex) [29]. When patients are diagnosed with mixed dysfunction, more attention should be paid to their emotional status, which is usually ignored in clinical practice. Additionally, it can be assumed that enhancement of chemosensory input, both taste and smell, may serve as a supplementary therapeutic strategy to relieve depressive symptoms.

Our results showed that patients with anosmia had higher depression scores than patients with hyposmia and controls. Depression scores in patients with hyposmia were also higher than those of controls, although this difference did not reach statistical significance. Additionally, both in the correlation and regression analysis, the TDI score was negatively associated with the BDI score in patients with chemosensory disorders. Nevertheless, it should be noted that the coefficients of correlation between olfactory scores and depression scores were relatively weak. Connections between the chemosensory system and emotions, which might serve as explanations for the currently observed associations, can be found through at least 2 mechanisms. First, olfactory impairment may lead to restrictions in olfactory-related areas, which can affect QoL, which in turn may enhance depression likelihood. Second, olfactory loss may reduce input from the olfactory bulb via the amygdala into the limbic system and insula and then cause dysfunction of convergence and salience processes, resulting in functional abnormalities of the brain [1]. Therefore, it is possible that there are many confounding factors between olfaction and depression in patients with chemosensory disorders, which may lead to the (weak) association between the 2 systems. Conversely, a twofold mechanism is assumed about the effect of depression on olfaction. On the one hand, reduced attention and reduced turnover rate of olfactory receptor neurons in the olfactory epithelium may cause reduced olfactory function in depression. These effects are reduced after remission of depression. On the other hand, decreased volume of olfactory bulb in depression patients may lead to reduced signaling from $\mathrm{OB}$ to central olfactory areas and exacerbate depressive symptoms [14]. Even though the relation between olfaction and depression is consistently observed in research and in the clinic, research has not been able to disentangle consequences from causes within this relation. Clinically, it is important to emphasize the usefulness of assessing chemosensory function in patients who might need emotional intervention. Further studies with large sample sizes and longer follow-up periods should be carried out to explore whether depression status may serve as a predictor of successful therapy.

Because most patients with chemosensory disorders exhibited mild depressive symptoms in the present study, their mean BDI scores were close to 10 and slightly high- 
er than those of the controls. It has been proven that olfactory dysfunction is not easy to recognize and can be compensated by other senses and cognition [37, 38]. Therefore, many hyposmic patients can still live a normal life, and only those with severe olfactory dysfunction exhibited obvious depression, which was consistent with the present correlation between BDI and TDI scores, as well as the results of comparison among controls, hyposmia, and anosmia. In summary, olfactory dysfunction exhibited a mild effect on depression, but attention still needs to be paid to those with moderate and severe depressive symptoms.

Previous studies $[25,39]$ suggested that gustation may be associated with depressive states, and Han et al. [40] found that patients with taste loss, but not patients with smell loss, exhibited a higher degree of depression than controls. However, we did not find a significant correlation between depression scores and taste scores, neither in the correlation analysis nor in the regression analysis. It must be kept in mind that within the current study, only suprathreshold gustatory testing was used, which limits the resolution of the characterization of taste dysfunctions. In addition, many patients with taste dysfunctions exhibit qualitative taste dysfunctions, which are poorly reflected in quantitative gustatory tests. Again, further studies with large sample sizes and more detailed taste tests, such as the "taste strips" [41] or gustatory event-related potentials [42], should be carried out to further clarify the relationship between taste and depression.

In the current study, chemosensory disorders caused by trauma were associated with poorer olfactory function and more severe symptoms of depression compared to other causes. Depression is known to be one of the most common comorbidities developing post-injury, and the frequency of depressive disorders in previous studies ranged widely (6-77\%) owing to methodological heterogeneity [43]. Chemosensory disorders caused by trauma are typically accompanied by damage to the central nervous system, especially in the olfactory bulb and frontal lobe [44, 45]. As mentioned above, the olfactory system is strongly interconnected with the emotional system, and both the olfactory bulb and frontal lobe play important roles in the regulation of emotion [17]. Hence, the accompanying damage to odor-related brain areas may be the underlying reason for depressive symptoms. On the other hand, it may be possible that other causes of chemosensory disorders exert their damage in the "periphery" of the olfactory pathway at the level of the olfactory epithelium and the olfactory bulb, resulting in mild- er olfactory impairment and fewer depressive symptoms than traumatic patients. Neuroimaging studies could help clarify the relationship between depressive symptoms and brain damage in trauma patients. Notably, damage to odor-related brain areas could also occur in chemosensory disorders caused by neurodegenerative diseases, leading to dysfunction of emotional processing and depressive symptoms [17]. Unfortunately, in the current study, the sample size of the neurodegeneration group was too small $(n=8)$ to be analyzed in a meaningful way.

The current study has several limitations. First, it should be noted that the current study focused on depressive symptoms rather than major depression disorder. Depression was assessed by the BDI, a self-rated scale that can only assess the severity of depression symptoms. The diagnosis of major depression and clinical interviews evaluated by a psychiatrist was lacking. Second, there is an imbalance of sample size regarding the distinct patient groups, which may have affected the statistical analyses. For example, the number of patients with taste dysfunctions and mixed dysfunctions was much smaller than that of patients with smell dysfunction, preventing the regression from being performed in these subgroups. Third, the lack of a linear correlation between depression and gustatory function may be influenced by the choice of taste test because the taste test used in the current study may have been too coarse to reflect the patients' gustatory abilities in full detail (gustatory threshold, discrimination, and identification). More reliable taste tests, such as the "taste strips" [46] or gustatory event-related potentials [47], should be used in the future to explore the relationship between taste and depression. Fourth, although age was included as a covariate in the statistical analysis, the smaller sample size and the younger age of controls versus patients may influence the results. Therefore, the present results should be interpreted with caution. Finally, the present results originated from a retrospective study design, and future follow-up studies could provide a deeper understanding of the relationship between chemosensory disorders and depression.

\section{Conclusion}

The present study suggests that simultaneous decreases in olfactory and gustatory sensations exacerbate emotional dysfunction. More attention should be paid to symptoms of depression in patients with mixed olfactory/ gustatory dysfunction in clinical practice. 


\section{Statement of Ethics}

This retrospective study was conducted in accordance with the guidelines of the Declaration of Helsinki, the retrospective protocol was approved by the Ethics Committee at the TU Dresden (protocol number EK251112006). As this is a retrospective study and patient data was completely anonymized/de-identified, informed consent was not obtained.

\section{Conflict of Interest Statement}

The authors have no conflicts of interest to declare.

\section{Funding Sources}

Deutsche Forschungsgemeinschaft DFG HU411/18-1.

\section{Author Contributions}

Ben Chen: conception and design, acquisition of data, analysis and interpretation of data; drafted the article; and gave final approval of the version to be published. Cara Benzien: conception and design, acquisition of data, and gave final approval of the version to be published. Vanda Faria: revised the manuscript critically for important intellectual content and gave final approval of the version to be published. Yuping Ning: revised the manuscript critically for important intellectual content and gave final approval of the version to be published. Mandy Cuevas: acquisition of data and gave final approval of the version to be published. Jana Linke: acquisition of data and gave final approval of the version to be published. Ilona Croy: revised the manuscript critically for important intellectual content and gave final approval of the version to be published. Antje Haehner: revised the manuscript critically for important intellectual content and gave final approval of the version to be published. Thomas Hummel: conception and design, acquisition of data, analysis and interpretation of data; revised the manuscript critically for important intellectual content, and gave final approval of the version to be published.

\section{References}

1 Croy I, Nordin S, Hummel T. Olfactory disorders and quality of life-an updated review. Chem Senses. 2014;39(3):185-94.

2 Maheswaran T, Abikshyeet P, Sitra G, Gokulanathan S, Vaithiyanadane V, Jeelani S. Gustatory dysfunction. J Pharm Bioallied Sci. 2014;6(Suppl 1):S30.

3 Croy I, Negoias S, Novakova L, Landis BN, Hummel T. Learning about the functions of the olfactory system from people without a sense of smell. Plos One. 2012;7(3):e33365.

4 Smeets MA, Veldhuizen MG, Galle S, Gouweloos J, de Haan AM, Vernooij J, et al. Sense of smell disorder and health-related quality of life. Rehabil Psychol. 2009;54(4):404-12.

5 Temmel AF, Quint C, Schickinger-Fischer B, Klimek L, Stoller E, Hummel T. Characteristics of olfactory disorders in relation to major causes of olfactory loss. Arch Otolaryngol Head Neck Surg. 2002;128(6):635-41.

6 Simopoulos E, Katotomichelakis M, Gouveris H, Tripsianis G, Livaditis M, Danielides V. Olfaction-associated quality of life in chronic rhinosinusitis: adaptation and validation of an olfaction-specific questionnaire. Laryngoscope. 2012;122(7):1450-4

7 Boesveldt S, Lindau ST, Mcclintock MK, Hummel T, Lundstrom JN, Lindstrom JN. Gustatory and olfactory dysfunction in older adults: a national probability study. Rhinology. 2011;49(3):324-30.

8 Seo HS, Jeon KJ, Hummel T, Min BC. Influences of olfactory impairment on depression, cognitive performance, and quality of life in Korean elderly. Eur Arch Otorhinolaryngol. 2009;266(11):1739.

9 Gopinath B, Anstey KJ, Kifley A, Mitchell P. Olfactory impairment is associated with functional disability and reduced independence among older adults. Maturitas. 2012;72(1): 50-5.

10 Walliczek-Dworschak U, Schöps F, Feron G, Brignot H, Hähner A, Hummel T. Differences in the density of fungiform papillae and composition of saliva in patients with taste disorders compared to healthy controls. Chem Senses. 2017;42(8):699.

11 Deems DA, Doty RL, Settle RG, Moore-Gillon V, Shaman P, Mester AF, et al. Smell and taste disorders, a study of 750 patients from the University of Pennsylvania smell and taste center. Arch Otolaryngol Head Neck Surg. 1991;117(5):519.

12 Merkonidis C, Grosse F, Ninh T, Hummel C, Haehner A, Hummel T. Characteristics of chemosensory disorders-results from a survey. Eur Arch Otorhinolaryngol. 2015;272(6):1403-16.

13 Chen B, Zhong X, Mai N, Peng Q, Zhang M, Chen $\mathrm{X}$, et al. Interactive effect of depression and cognitive impairment on olfactory identification in elderly people. JAD. 2018;66(4): 1645-55.

14 Croy I, Symmank A, Schellong J, Hummel C, Gerber J, Joraschky P, et al. Olfaction as a marker for depression in humans. J Affect Disord. 2016;160:80-6.

15 Yuan TF, Slotnick BM. Roles of olfactory system dysfunction in depression. Prog Neuropsychopharmacol Biol Psychiatry. 2014;54: 26-30.

16 Negoias S, Croy I, Gerber J, Puschmann S, Petrowski K, Joraschky P, et al. Reduced olfactory bulb volume and olfactory sensitivity in patients with acute major depression. Neuroscience. 2010;169(1):415-21.

17 Marine N, Boriana A. Olfactory markers of depression and Alzheimer's disease. Neurosci Biobehav Rev. 2014;45:262-70.
18 Khil L, Rahe C, Wellmann J, Baune BT, Wersching $\mathrm{H}$, Berger K. Association between major depressive disorder and odor identification impairment. J Affect Disord. 2016;203: 332-8.

19 Preeti K, Soler ZM, Nguyen SA, Muus JS, Schlosser RJ. The association between olfaction and depression: a systematic review. Chem Senses. 2016;6.

20 Taalman H, Wallace C, Milev R. Olfactory functioning and depression: a systematic review. Front Psychiatry. 2017;8:190.

21 Rottstaedt F, Weidner K, Strauss T, Schellong J, Kitzler H, Wolff-Stephan S, et al. Size matters - the olfactory bulb as a marker for depression. J Affect Disord. 2018;229:193-8.

22 Croy I, Symmank A, Schellong J, Hummel C, Gerber J, Joraschky P, et al. Olfaction as a marker for depression in humans. J Affect Disord. 2014;160:80-6.

23 Birte-Antina W, Ilona C, Antje $\mathrm{H}$, Thomas $\mathrm{H}$. Olfactory training with older people. Int J Geriatr Psychiatry. 2018;33(1):212-20.

24 Komori T, Fujiwara R, Tanida M, Nomura J, Yokoyama MM. Effects of citrus fragrance on immune function and depressive states. Neuroimmunomodulation. 1995;2(3):17480.

25 Andersen SB, Mcmahon B, Madsen MK, Knudsen GM, Holst KK, Møller P, et al. Sweet taste sensitivity is influenced by 5 -HTTLPR genotype and affected in seasonal affective disorder. Psychiatry Res. 2014;220(1-2): 727-9.

26 Swiecicki L, Scinska A, Bzinkowska D, Torbinski J, Sienkiewicz-Jarosz H, Samochowiec J, et al. Intensity and pleasantness of sucrose taste in patients with winter depression. Nutr Neurosci. 2015;18(4):186-91. 
27 Chen D, Dalton P. The effect of emotion and personality on olfactory perception. Chem Senses. 2005;30(4):345-51.

28 Pollatos O, Kopietz R, Linn J, Albrecht J, Sakar V, Anzinger A, et al. Emotional stimulation alters olfactory sensitivity and odor judgment. Chem Senses. 2007;32(6):583-9.

29 Khil L, Wellmann J, Berger K. Impact of combined sensory impairments on health-related quality of life. Qual Life Res. 2015;24(9): 2099-103.

30 Hummel T, Whitcroft KL, Andrews P, Altundag A, Cinghi C, Costanzo RM, et al. Position paper on olfactory dysfunction. Rhinology. 2017;56(1):1-30.

31 Beck AT, Steer RA, Carbin MG. Psychometric properties of the beck depression inventory: twenty-five years of evaluation. Clin Psychol Rev. 1988;8(1):77-100.

32 Hummel T, Hummel C, Welge-Luessen A. Assessment of olfaction and gustation. In: Welge-Luessen A, Hummel T, editors. Management of smell and taste disorders: a practical guide for clinicians. Stuttgart: Thieme; 2013. p. $58-75$

33 Kobal G, Hummel T, Sekinger B, Barz S, Roscher S, Wolf S. "Sniffin' sticks": screening of olfactory performance. Rhinology. 1996; 34:222.

34 Hummel T, Kobal G, Gudziol H, Mackay-Sim A. Normative data for the "Sniffin' Sticks" in- cluding tests of odor identification, odor discrimination, and olfactory thresholds: an upgrade based on a group of more than 3,000 subjects. Eur Arch Otorhinolaryngol. 2007; 264:237-43.

35 Oleszkiewicz A, Schriever VA, Croy I, Hähner A, Hummel T. Updated Sniffin' Sticks normative data based on an extended sample of 9,139 subjects. Eur Arch Otorhinolaryngol. 2019;276(3):719-28.

36 Faulcon P, Portier F, Biacabe B, Bonfils P. Anosmia secondary to acute rhinitis: clinical signs and course in a series of 118 patients. Ann Otolaryngol Chir Cervicofac. 1999;116:351-7.

37 Oleszkiewicz A, Hummel T. Whose nose does not know? Demographical characterization of people unaware of anosmia. Eur Arch Otorhinolaryngol. 2019;276(6):1849-52.

38 Yoo HS, Chung SJ, Lee YH, Ye BS, Sohn YH, Lee PH. Olfactory anosognosia is a predictor of cognitive decline and dementia conversion in Parkinson's disease. J Neurol. 2019;266(7): 1601-10.

39 Nagai M, Matsumoto S, Endo J, Sakamoto R, Wada M. Sweet taste threshold for sucrose inversely correlates with depression symptoms in female college students in the luteal phase. Physiol Behav. 2015;141:92-6.

40 Han P, Georgi M, Cuevas M, Haehner A, Gudziol V, Hummel T. Decreased electrogustometric taste sensitivity in patients with ac- quired olfactory dysfunction. Rhinology. 2018;56(2):158-65.

41 Wolf A, Varga L, Wittibschlager L, Renner B, Mueller CA. A self-administered test of taste function using "Taste Strips". Int Forum Allergy Rhinol. 2016;6(4):362-6.

42 Schienle A, Giraldo M, Spiegl B, Schwab D. Influence of bitter taste on affective facial processing: an ERP study. Chem Senses. 2017; 42(6):473-8

43 Jones M, Acion L, Jorge RE. What are the complications and emerging strategies for preventing depression following traumatic brain injury? Expert Rev Neurother. 2017; 17(6):631-40.

44 Singh R, Humphries T, Mason S, Lecky F, Dawson J, Sinha S. The incidence of anosmia after traumatic brain injury: the SHEFBIT cohort. Brain Inj. 2018;32(9):1122-8.

45 Coelho DH, Costanzo RM. Posttraumatic olfactory dysfunction. Auris Nasus Larynx. 2016;43(2):137-43.

46 Mueller C, Kallert S, Renner B, Stiassny K, Temmel AF, Hummel T, et al. Quantitative assessment of gustatory function in a clinical context using impregnated "taste strips". Rhinology. 2003;41(1):2-6.

47 Iannilli E, Broy F, Kunz S, Hummel T. Agerelated changes of gustatory function depend on alteration of neuronal circuits. J Neurosci Res. 2017;95(10):1927. 